morphologically. As mentioned above, such chemotaxonomic considerations would today raise a red flag against halogeton. Another example is the sicklepod milkvetch (Astragalus falcatus Lam.), which was introduced around 1920 and has been recommended for range plantings for over 25 years; recognition that many Astragalus species contain toxic concentrations of nitro compounds led to identification of sicklepod milkvetch as poisonous, and efforts are now being directed to its eradication. Fourth, introduced plants that are suspected of being poisonous may be fed to experimental animals, as extracts, dried plants, or residues.

With current pressures to strip-mine for coal, there is much interest in finding plant species that will grow on mine spoils, reproducing rapidly and providing erosion control, and, if possible, grazing for livestock and food and cover for wildlife. The clear need for such plants to be nonpoisonous gives special urgency to the programme outlined above.

\title{
Geomagnetic field modelling using satellite data
}

\section{from David R. Barraclough}

ACCURATE DESCRIPTIONS of the Earth's magnetic field are useful for navigational purposes and form the basis for many scientific studies. Such descriptions usually take the form of mathematical models, most often in terms of an expansion in spherical harmonics, and magnetic charts can readily be produced from the models. A major difficulty in producing geomagnetic field models has always been the acquisition of an up-to-date and well distributed set of accurate observations. As a satellite in a polar orbit can survey the entire globe in a matter of days, it was an obvious step to use such a satellite to measure the geomagnetic field.

The most recent satellite survey of the geomagnetic field was performed by the NASA/US Geological Survey satellite Magsat between November 1979 and June 1980. Magsat differed from previous geomagnetic survey satellites in that it was able to measure not only the strength of the field but also its direction.

Since the geomagnetic field is derivable from a scalar potential which satisfies Laplace's equation, it was possible to use the earlier satellite observations of $F$ to refine existing models of the field. Although such models fitted the input $F$ data very well (with a r.m.s. residual of about $10 \mathrm{nT}$ ), there was evidence that they did not describe the vector geomagnetic field as accurately (Ben'kova et al. Bull. int. Ass. Geomag. Aeron. No. 152-163; 1971; Fabiano \& Peddie J. geophys. Res. $76,3816 ; 1971)$. These findings led to several theoretical studies of the production of field models from $F$ observations alone.

Hurwitz and Knapp (J. geophys. Res. 79,$3009 ; 1974$ ) and Barraclough and Nevitt (Phys. Earth planet. Interiors 13, 123; 1976), using simulated data with noise added, verified that good fits to $F$ did not

David R. Barraclough is in the Geomagnetism Unit, Institute of Geological Sciences, Edinburgh. guarantee a good description of the other field components. In particular, large errors in the vertical component $(Z)$ of the field occurred near the dip-equator (the line of zero inclination). In terms of the coefficients of the spherical harmonic model, those having equal values of $m$ and $n$ were least well determined. These are the sectorial harmonics, which divide the surface of a sphere into segments like those of an orange. It was also shown that the large errors could be removed by using small amounts of vector data (about 10 per cent of the total) covering the equatorial region.

Hurwitz and Knapp (op. cit.) and Lowes (Geophys. J. R. astr. Soc. 42, 637; 1975) showed that the root of the problem lay with what they termed the perpendicular error effect. When fitting only a single component of a vector field, the perpendicular components synthesized from the resulting model can have errors associated with them which are considerably larger than the errors in the component fitted. This results from the lack of constraints in the perpendicular directions. In some cases, for example the production of models from $Z$ data only, the perpendicular error effect is of minor importance because of the particular configuration of the geomagnetic field. It turns out, however, that fitting $F$ data is a particularly bad choice. The perpendicular error effect leads to large errors in $Z$ and these are especially troublesome where $Z$ is small, that is, near the dip-equator, as noted above.

Backus (Q. Jl Mech. appl. Math. 21, 195; 1968; J. geophys. Res. 75, 6339; 1970; Geophys. Res. Lett. 1, 21; 1974) investigated whether models derived from $F$ data alone were unique and showed that, under certain conditions, they were not. In practical field modelling using real data these conditions for non-uniqueness are not fulfilled. Stern and Bredekamp ( $J$. geophys. Res. 80, 1776; 1975) have, however, shown that certain series of terms in the spherical harmonic expansion, which Backus highlighted in his uniqueness studies, are likely to be relatively poorly determined in an analysis of $F$ data alone. These series (which Stern and Bredekamp called 'Backus series') consist of terms which all have the same value of $m$. Each series starts with a sectional $(n=m)$ term and succeeding terms have values of $n$ equal to $m+2, m+4, m+6$, and so on. In agreement with the findings noted above, Stern and Bredekamp found, using simulated data, that the terms with $m=n$ had the largest uncertainty for a given series.

Now that data are available from Magsat, it is possible to study these effects using real data. Stern et al. (Geophys. Res. Lett. 7,$941 ; 1980$ ), in one of the first reports of results from Magsat, have made an initial attempt at this. They have compared two models, one based on vector data from early in the Magsat mission (November 1979) and the other based only on scalar $(F)$ data collected at about the same time. Values of $Z$ computed from the two models differed by up to $2,300 \mathrm{nT}$ at the Earth's surface and by up to 1,500 nT at the satellite's altitude. (For comparison, typical mid-latitude values of $Z$ are of the order of 40,000 nT.) The differences, when plotted on a world map, showed a pattern of six foci, all positioned very near to the dip-equator. When the two models were compared coefficient by coefficient, those in the Backus series described above did indeed show larger differences than the coefficients not belonging to such series. There were suggestions that other effects were present affecting the 'non-Backus' terms and work is in progress in this area.

Thus, the doubts cast on geomagnetic field models based solely on $F$ data seem to be confirmed. Fortunately there are relatively few such models and most of these have been produced for specific purposes related to the scalar data on which they were based. Most producers of models for navigational and general scientific purposes (for example, Barraclough et al. Geophys. J. R. astr. Soc. 43, 645; 1975; Barker et al. Geophys. J. R. astr. Soc. in the press) have been careful to use $F$ observations as only a part of the input data and their models will therefore be free from the deficiencies discussed above. Potential users of field models should be wary in their choice of models. Field models based solely on $F$ data can be used with some confidence if one is interested only in the intensity of the geomagnetic field. If vector information is needed it is best to avoid such models and to use instead one of the models based on component data as well as $F$ data. The International Association of Geomagnetism and Aeronomy, at its next scientific assembly this summer, plans to recommend an International Geomagnetic Reference Field which should provide a set of reliable models describing the geomagnetic field since 1965 . 